\title{
On Terai's conjecture concerning Pythagorean numbers
}

\author{
by \\ MaOhua Le (Zhanjiang)
}

1. Introduction. Let $\mathbb{Z}, \mathbb{N}, \mathbb{Q}$ be the sets of integers, positive integers and rational numbers respectively. Let $(a, b, c)$ be a primitive Pythagorean triple such that

$$
a^{2}+b^{2}=c^{2}, \quad a, b, c \in \mathbb{N}, \quad \operatorname{gcd}(a, b, c)=1, \quad 2 \mid a .
$$

Then we have

$$
a=2 u v, \quad b=u^{2}-v^{2}, \quad c=u^{2}+v^{2},
$$

where $u, v$ are positive integers satisfying

$$
u>v, \quad \operatorname{gcd}(u, v)=1, \quad 2 \mid u v .
$$

In [7], Terai conjectured that the equation

$$
x^{2}+b^{y}=c^{z}, \quad x, y, z \in \mathbb{N},
$$

has only the solution $(x, y, z)=(a, 2,2)$. This conjecture was proved for some special cases. But, in general, the problem is far from solved. In this respect, the author $[3]$ proved that if $b>8 \cdot 10^{6}, b \equiv \pm 5(\bmod 8)$ and $c$ is a prime power, then (4) has only the solution $(x, y, z)=(a, 2,2)$. Afterwards, Cao and Dong [1], Yuan [8] showed that the condition $b>8 \cdot 10^{6}$ can be eliminated from the result of [3]. In addition, Cao and Dong [1], Yuan and Wang [9] proved that if $b \equiv \pm 5(\bmod 8)$ and either $b$ or $c$ is a prime, then (4) has only the solution $(x, y, z)=(a, 2,2)$. In this paper we consider the case of $b \not \equiv \pm 5(\bmod 8)$. We prove the following result.

TheOREM. If $b \equiv 7(\bmod 8)$ and either $b$ is a prime or $c$ is a prime power, then (4) has only the solution $(x, y, z)=(a, 2,2)$.

2000 Mathematics Subject Classification: Primary 11D61.

Supported by the National Natural Science Foundation of China, the Guangdong Provincial Natural Science Foundation and the Natural Science Foundation of the Education Department of Guangdong Province. 


\section{Preliminaries}

Lemma 1 ([5, pp. 12-13]). Every solution $(X, Y, Z)$ of the equation

$$
X^{2}+Y^{2}=Z^{2}, \quad X, Y, Z \in \mathbb{N}, \quad \operatorname{gcd}(X, Y)=1, \quad 2 \mid X,
$$

can be expressed as

$$
X=2 r s, \quad Y=r^{2}-s^{2}, \quad Z=r^{2}+s^{2},
$$

where $r, s$ are positive integers satisfying

$$
r>s, \quad \operatorname{gcd}(r, s)=1, \quad 2 \mid r s .
$$

Lemma 2 ([5, pp. 122-123]). Let $n$ be an odd integer with $n \geq 1$. Every solution $(X, Y, Z)$ of the equation

$$
X^{2}+Y^{2}=Z^{n}, \quad X, Y, Z \in \mathbb{Z}, \quad \operatorname{gcd}(X, Y)=1,
$$

can be expressed as

$$
Z=r^{2}+s^{2}, \quad X+Y \sqrt{-1}=\lambda_{1}\left(r+\lambda_{2} s \sqrt{-1}\right)^{n}, \quad \lambda_{1}, \lambda_{2} \in\{1,-1\},
$$

where $r, s$ are positive integers satisfying (5).

Lemma 3 ([5, Theorem 4.2]). The equation

$$
X^{2}+Y^{4}=Z^{4}, \quad X, Y, Z \in \mathbb{N}, \quad \operatorname{gcd}(X, Y)=1,
$$

has no solution $(X, Y, Z)$.

Lemma 4 ([4] and $[6])$. The equation

$$
1+X^{2}=2 Y^{n}, \quad X, Y, n \in \mathbb{N}, X>1, Y>1, n>2,
$$

has only the solution $(X, Y, n)=(239,13,4)$.

Lemma 5 ([2, Lemma 1]). Let $D$ be a positive integer, and let $p$ be an odd prime with $p \nmid D$. If the equation

$$
X^{2}+D Y^{2}=p^{Z}, \quad X, Y, Z \in \mathbb{Z}, \quad \operatorname{gcd}(X, Y)=1, \quad Z>0,
$$

has a solution $(X, Y, Z)$, then it has a unique solution $\left(X_{1}, Y_{1}, Z_{1}\right)$ such that $X_{1}>0, Y_{1}>0$ and $Z_{1} \leq Z$, where $Z$ runs through all solutions $(X, Y, Z)$ of (6). $\left(X_{1}, Y_{1}, Z_{1}\right)$ is called the least solution of (6). Moreover, every solution $(X, Y, Z)$ of $(6)$ can be expressed as

$Z=Z_{1} t, \quad X+Y \sqrt{-D}=\lambda_{1}\left(X_{1}+\lambda_{2} Y_{1} \sqrt{-D}\right)^{t}, \quad t \in \mathbb{N}, \lambda_{1}, \lambda_{2} \in\{-1,1\}$.

3. Proof of Theorem. Let $(x, y, z)$ be a solution of (4) with $(x, y, z) \neq$ $(a, 2,2)$. Since $b \equiv 7(\bmod 8)$, we see from $(2),(3)$ and $(4)$ that $c \equiv 1(\bmod 8)$ and $2 \mid y$.

We first consider the case that $2 \mid y$ and $2 \mid z$. By Lemma 1 , from (4) we then get

$$
x=2 r s, \quad b^{y / 2}=r^{2}-s^{2}, \quad c^{z / 2}=r^{2}+s^{2},
$$


where $r, s$ are positive integers satisfying (5). Since $(x, y, z) \neq(a, 2,2)$, if $y=2$, then $z>2$ and $z / 2 \geq 2$. By (4) and (7), we get

$$
r^{2}+s^{2}=c^{z / 2} \geq c^{2}>b^{2}=\left(r^{2}-s^{2}\right)^{2} \geq(r+s)^{2}>r^{2}+s^{2},
$$

a contradiction. Similarly, if $z=2$, then $y>2$ and $y / 2 \geq 2$. Hence, we deduce from (2) and (4) that

$$
\begin{aligned}
u^{2}+v^{2} & =c=c^{z / 2}=\sqrt{x^{2}+b^{y}}>b^{y / 2} \\
& \geq b^{2}=\left(u^{2}-v^{2}\right)^{2} \geq(u+v)^{2}>u^{2}+v^{2},
\end{aligned}
$$

a contradiction. So we have $y>2$ and $z>2$.

If $b$ is a prime, then from (7) we get $r=s+1, b^{y / 2}=2 s+1$ and $c^{z / 2}=2 s^{2}+2 s+1$. This implies that

$$
1+b^{y}=2 c^{z / 2} .
$$

Since $2 \mid y$, by Lemma 4 , we find from (10) that either $z / 2=2$ or $(b, c, y, z)=$ $(239,13,2,8)$. When $z / 2=2$, by Lemma 3 , we see from (2), (4) and (10) that $y \geq 6$ and

$$
\begin{aligned}
2 u^{4}+4 u^{2} v^{2}+2 v^{4} & =2\left(u^{2}+v^{2}\right)^{2}=2 c^{2}=2 c^{z / 2} \\
& >b^{y} \geq b^{6}=\left(u^{2}-v^{2}\right)^{6} \\
& \geq(u+v)^{6}>4 u^{4}+6 u^{2} v^{2}+4 v^{4},
\end{aligned}
$$

a contradiction. When $(b, c)=(239,13), b$ and $c$ do not satisfy (1). Thus, the Theorem holds for this case.

If $c$ is a prime power, then

$$
c=p^{k},
$$

where $p$ is an odd prime and $k$ is a positive integer. We see from (1), (4) and (12) that the equation

$$
X^{2}+b^{2} Y^{2}=p^{Z}, \quad X, Y, Z \in \mathbb{Z}, \quad \operatorname{gcd}(X, Y)=1, \quad Z>0,
$$

has two solutions $(X, Y, Z)=(a, 1,2 k)$ and $\left(x, b^{(y-2) / 2}, z k\right)$. Let $\left(X_{1}, Y_{1}, Z_{1}\right)$ be the least solution of (13). By Lemma 5 , if $\left(X_{1}, Y_{1}, Z_{1}\right) \neq(a, 1,2 k)$, then we have

$$
\begin{gathered}
2 k=Z_{1} t, \quad t \in \mathbb{N}, t>1, \\
a+\sqrt{-b^{2}}=\lambda_{1}\left(X_{1}+\lambda_{2} Y_{1} \sqrt{-b^{2}}\right)^{t}, \quad \lambda_{1}, \lambda_{2} \in\{-1,1\} .
\end{gathered}
$$

By (15), we get $2 \nmid t$. So we have $t \geq 3$. Since $X_{1}^{2}+b^{2} Y_{1}^{2}=p^{Z_{1}}$, we infer from (2) and (12) that

$$
\begin{aligned}
u^{2}+v^{2} & =c=p^{Z_{1} t / 2} \geq p^{3 Z_{1} / 2}=\left(X_{1}^{2}+b^{2} Y_{1}^{2}\right)^{3 / 2}>b^{3}=\left(u^{2}-v^{2}\right)^{3} \\
& \geq(u+v)^{3}>u^{3}+v^{3}
\end{aligned}
$$


a contradiction. This implies that $\left(X_{1}, Y_{1}, Z_{1}\right)=(a, 1,2 k)$. Using Lemma 5 again, we get

$$
\begin{gathered}
z k=2 k t, \quad t \in \mathbb{N}, t>1, \\
x+b^{(y-2) / 2} \sqrt{-b^{2}}=\lambda_{1}\left(a+\lambda_{2} \sqrt{-b^{2}}\right)^{t}, \quad \lambda_{1}, \lambda_{2} \in\{-1,1\} .
\end{gathered}
$$

Since $2 \nmid b$, we find from (18) that $2 \nmid t$ and

$$
b^{(y-2) / 2}=\lambda_{1} \lambda_{2} \sum_{i=0}^{(t-1) / 2}\left(\begin{array}{c}
t \\
2 i+1
\end{array}\right) a^{t-2 i-1}\left(-b^{2}\right)^{i} .
$$

Since $\operatorname{gcd}(a, b)=1$ and $y>2$, we see from (19) that $b \mid t$. Further, using the same method as in the proof of [3, Theorem], we can deduce from (19) that $b^{(y-2) / 2} \mid t$. So we have $t \geq b^{(y-2) / 2}$. Therefore, by (7), (12) and (17), we obtain

$$
\begin{aligned}
b^{y} & =\left(r^{2}-s^{2}\right)^{2} \geq(r+s)^{2}>r^{2}+s^{2}=c^{z / 2} \\
& =p^{z k / 2}=p^{k t}=c^{t}>b^{t} \geq b^{b^{(y-2) / 2}},
\end{aligned}
$$

whence we get

$$
y>b^{(y-2) / 2} .
$$

However, since $y \geq 4$ and $b \geq 7,(21)$ is impossible. Thus, under the hypothesis, (4) has only the solution $(z, y, z)=(a, 2,2)$ satisfying $2 \mid y$ and $2 \mid z$.

We next consider the case that $2 \mid y$ and $2 \nmid z$. If $b$ is a prime, then from (2) we get

$$
u=v+1, \quad b=2 v+1, \quad c=2 v^{2}+2 v+1, \quad v \equiv 3(\bmod 4) .
$$

On the other hand, by Lemma 2, we see from (4) that

$$
\begin{gathered}
c=r^{2}+s^{2}, \\
x+b^{y / 2} \sqrt{-1}=\lambda_{1}\left(r+\lambda_{2} s \sqrt{-1}\right)^{z}, \quad \lambda_{1}, \lambda_{2} \in\{-1,1\},
\end{gathered}
$$

where $r, s$ are positive integers satisfying (5). From (24), we get

$$
b^{y / 2}=\lambda_{1} \lambda_{2} s \sum_{i=0}^{(z-1) / 2}\left(\begin{array}{c}
z \\
2 i+1
\end{array}\right) r^{z-2 i-1}\left(-s^{2}\right)^{i} .
$$

We see from (25) that $s$ satisfies either $s=1$ or $b \mid s$. When $s=1$, we infer from $(22)$ and $(23)$ that $r^{2}=2 v(v+1)$. This implies that $v$ is a square with $v \equiv 3(\bmod 4)$, which is a contradiction. When $b \mid s$, we have $s \geq b$. Hence, by $(22)$ and (23), we get

$$
2 v^{2}+2 v+1=c=r^{2}+s^{2}>s^{2} \geq b^{2}=(2 v+1)^{2}=4 v^{2}+4 v+1,
$$

a contradiction. 
If $c$ is a prime power, then $c$ can be expressed as (12). Moreover, by the above analysis, $(13)$ then has two solutions $(X, Y, Z)=(a, 1,2 k)$ and $\left(x, b^{(y-2) / 2}, z k\right),\left(X_{1}, Y_{1}, Z_{1}\right)=(a, 1,2 k)$ is the least solution of $(13)$ and $z$ satisfies (17). So we have $z=2 t$ and $z$ is even, a contradiction. Thus, under the hypothesis, (4) has no solution $(x, y, z)$ satisfying $2 \mid y$ and $2 \nmid z$. To sum up, the Theorem is proved.

\section{References}

[1] Z. F. Cao and X. L. Dong, On Terai's conjecture, Proc. Japan Acad. Ser. A Math. Sci. 74 (1998), 127-129.

[2] M. H. Le, On the number of solutions of the diophantine equation $x^{2}+D=p^{n}$, C. R. Acad. Sci. Paris Sér. I Math. 317 (1993), 135-138.

[3] - A note on the diophantine equation $x^{2}+b^{y}=c^{z}$, Acta Arith. 71 (1995), 253-257.

[4] W. Ljunggren, Zur Theorie der Gleichung $x^{2}+1=D y^{4}$, Avh. Norske Vid. Akad. Oslo 5 (1942), 1-27.

[5] L. J. Mordell, Diophantine Equations, Academic Press, London, 1969.

[6] C. Störmer, L'équation $m \arctan 1 / x+n \arctan 1 / y=k \pi / 4$, Bull. Soc. Math. France 27 (1899), 160-170.

[7] N. Terai, The Diophantine equation $x^{2}+q^{m}=p^{n}$, Acta Arith. 63 (1993), 351-358.

[8] P. Z. Yuan, On the diophantine equation $x^{2}+b^{y}=c^{z}$, J. Sichuan Univ. Nat. Sci. Ser. 35 (1998), 5-7.

[9] P. Z. Yuan and J. B. Wang, On the diophantine equation $x^{2}+b^{y}=c^{z}$, Acta Arith. 84 (1998), 145-147.

Department of Mathematics

Zhanjiang Normal College

Postal Code 524048

Zhanjiang, Guangdong, P.R. China

Received on 14.3.2000

and in revised form on 23.4.2001 